\title{
Three-Dimensional Seismic Collapse Analysis of Wooden Houses Using Rigid Body-Spring Method
}

\author{
E. A. Tingatinga, H. Kawakami* and S.M. Shrestha \\ Geosphere Research Institute, Saitama University, Saitama, Japan
}

\begin{abstract}
In Japan, the past few decades revealed the vulnerability of wood-framed residential buildings to strong earthquakes. The Kobe earthquake in 1995 caused tremendous loss of lives resulting from the collapse and damage of such structures that significantly affected economic condition. This disaster motivated many researchers to study the mechanisms of collapse of engineering structures in order to prevent further loss of lives in the future. In this paper, an innovative methodology in simulating the dynamic response of wood-framed buildings, for purposes of seismic performance assessment and retrofitting, is presented. The proposed method, which can simulate inelastic behavior of structures, is capable of showing realistic progressive collapse mechanisms and accurate seismic response of structures. The sequence of analyses and results in the form of computer animations are used to help building owners gain a better understanding of the seismic performance of their buildings before and after the structural reinforcement. Applications to real wood-framed residential buildings were used to show the effectiveness of the methodology in seismic performance assessment as well as retrofit plan development.
\end{abstract}

\section{INTRODUCTION}

The evolution in computer hardware has significant impact on computing science and engineering design. Desktop and portable computers nowadays are operating at tremendous speed, have huge memory resources and even multiple processors, thereby permitting very fast computations never conceived before. Because of these advancements, several researches have been conducted to simulate various natural phenomena and to analyze problems that were previously infeasible.

In civil engineering, computer experiments utilizing multimedia and realistic computer animation reinforce, if not replace, traditional expensive experiments. Computer experiments offer several advantages such that they allow analysis of structures at full-scale, experiments may be performed at ideal conditions when necessary e.g. no air resistance or friction, and they are not subject to physical limitations inherent to experimental set-ups, e.g. maximum displacement/velocity of shaking tables, etc.

Efforts to simulate response of built structures to strong motion earthquakes attracted many researchers in the field of architecture and civil engineering. One of the most familiar accelerogram used is that of the Kobe earthquake in Japan which occurred in January 17, 1995 and caused enormous damage and destruction of structures and deaths in Kobe and in nearby areas. The disaster claimed more than 6,000 deaths, injured more than 35,000 people and caused about 10 trillion yen worth of damage. The death toll was reported to be mostly caused by collapse of buildings and breakdown of other civil engineering facilities. Investigation further revealed that most deaths were due to collapse of traditional

*Address correspondence to this author at the Geosphere Research Institute, Saitama University, Saitama, Japan; Tel/Fax: +81488583543; E-mail: kaw@kiban.civil.saitama-u.ac.jp wooden houses. This fact motivated structural and earthquake engineers to study the mechanisms of collapse of each wooden house and building in order to guarantee the safety of the general public in the occurrence of future earthquakes.

Analysis of the collapse of engineering structures subjected to strong ground motions or even blast loads, may be performed using a handful of methods. The popular finite element method allows modeling of structures at the material level, but its computational complexity has restricted its application to analysis of basic structural elements such as beams, columns, and joints. Finite element analysis of an entire structure was regarded to be too tedious and that dynamic analysis exhibiting strong nonlinearity and discontinuity requires a lot of computer power.

This study presents a three-dimensional simulation of structure collapse during strong motion earthquakes, such as the 1995 Kobe earthquake, using Rigid Body-Spring Method (RBSM). Various modes of collapse of a wooden structure modeled as an assembly of rigid bodies connected by inelastic links at their ends, will be presented. In modeling structural components, a link configuration is suggested to take into account structural damping and inelastic behavior.

The main objectives of this study are to understand the process in which wooden houses collapse during earthquakes and to identify and reinforce the weak point of the structure. Specifically, it aims to

1. simulate collapse mechanisms by modeling structural elements using rigid body-spring method implementing a configuration of inelastic links;

2. investigate how the collapse progresses from the local failures of the framing members; and

3. propose, with the use of an example, ways to reinforce a house to withstand strong ground shaking. 


\section{NONLINEAR ANALYSIS USING ASSEMBLY OF RIGID BODIES}

Originally proposed by Kawai [1], the basic approach of RBSM is to divide the given structure into appropriate number of rigid elements connected by spring systems. The displacements are completely described by the positions and rotations of the rigid bodies while the deformation energy of the structure is stored in the spring system.

In this paper, nonlinear analysis of structures will be carried out by introducing nonlinear springs to take into account large displacements and failure of structures during strong motion earthquakes.

\section{POSITION AND ORIENTATION OF BODIES $[2,3]$}

In rigid body assemblies, various coordinates systems must first be well understood (Fig. 1). A rigid body in space is positioned with respect to the inertial coordinate system OXYZ by a vector $\boldsymbol{r}$ attached to its mass center. Points that define the shape of the body or points where links are attached, say point $a$, are defined by a vector $\boldsymbol{s}$ in terms of local coordinates (with respect to Gxyz). If needed, the global coordinates of this point $\boldsymbol{S}$ are computed as

$$
\boldsymbol{S}=\boldsymbol{R} \boldsymbol{s}+\boldsymbol{r}
$$

where $\boldsymbol{R}$ is a rotation matrix that transforms coordinates from Gxyz frame to OXYZ frame.

Orientation of the body can also be described using Hamilton's quaternion $\boldsymbol{q}$ [3] so that Gxyz is obtained by rotating OXYZ about an axis $\boldsymbol{u}$ by an angle $\gamma$.

$$
\boldsymbol{q}=\left[\cos \frac{\gamma}{2}, \boldsymbol{u} \sin \frac{\gamma}{2}\right]
$$

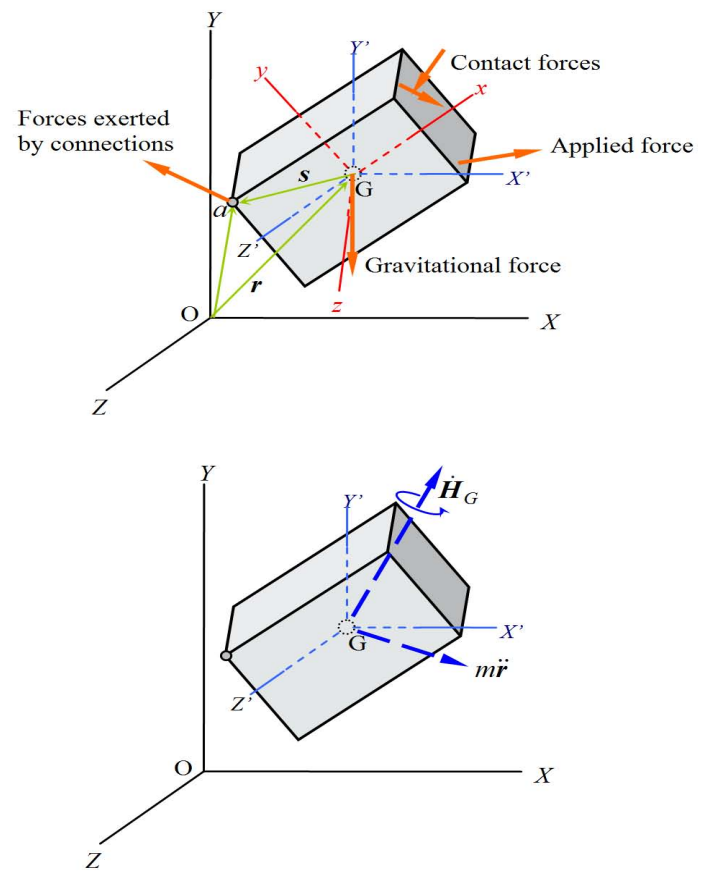

Fig. (1). Applied and effective forces on a rigid body and coordinate systems used in computer animation.

\section{GOVERNING EQUATIONS [2,3]}

To animate various systems using rigid bodies, appropriate forces must be taken into account. Forces that arise due to relative positioning of objects (e.g., contact or collision), object's velocity, connections (e.g., springs and dampers), and user-specified vector fields (e.g., gravity and other external forces) must be exerted on bodies properly. These forces induce linear and angular accelerations depending on the mass and mass distribution of the body, respectively.

The two fundamental equations used to analyze motions of rigid bodies in space are

$$
\begin{aligned}
& \sum \boldsymbol{F}(t)=m \ddot{\boldsymbol{r}}(t) \\
& \sum \boldsymbol{M}_{G}(t)=\dot{\boldsymbol{H}}_{G}(t)
\end{aligned}
$$

where $\ddot{\boldsymbol{r}}(t)$ is the acceleration of the center of mass and $\dot{\boldsymbol{H}}_{G}(t)$ is the rate of change of the angular momentum about the mass center of the rigid body. If $x, y$ and $z$ axes coincide with the principal axes of inertia of the rigid body, Eq. (4) can be expressed in terms of the inertia tensor $\boldsymbol{I}(t)$ as

$$
\sum \boldsymbol{M}_{G}(t)=\boldsymbol{I}(t) \dot{\omega}(t)
$$

The above equations can be rewritten by first assuming

$$
\begin{aligned}
& \dot{\boldsymbol{r}}(t)=\boldsymbol{v}(t) \\
& \dot{\boldsymbol{q}}(t)=\frac{1}{2} \boldsymbol{\omega}(t) * \boldsymbol{q}(t)=\boldsymbol{g}(\boldsymbol{q}(t), \boldsymbol{\omega}(t))
\end{aligned}
$$

where $\omega(t) * \boldsymbol{q}(t)$ denotes a shorthand of the multiplication of two quaternions $[0, \omega(t)]$ and $\boldsymbol{q}(t)$.

At any instant, the state of a rigid body is stored in a vector $\boldsymbol{x}(t)$ consisting of its position, orientation, and its linear and angular velocities (Baraff [2]). Mathematically collected as,

$$
\boldsymbol{x}(t)=\left\{\begin{array}{c}
\boldsymbol{r}(t) \\
\boldsymbol{q}(t) \\
\boldsymbol{v}(t) \\
\boldsymbol{\omega}(t)
\end{array}\right\}_{13 \times 1}
$$

The time derivative of the state vector can be set-up using Eqs. (3), (5), (6), and (7) as

$$
\dot{\boldsymbol{x}}(t)=\left\{\begin{array}{c}
\boldsymbol{v}(t) \\
\boldsymbol{g}(\boldsymbol{q}(t), \boldsymbol{\omega}(t)) \\
\sum \boldsymbol{F}(t) / m \\
\boldsymbol{I}^{-1}(t) \sum \boldsymbol{M}_{G}(t)
\end{array}\right\}
$$


This system of first-order differential equations are sufficient to perform physically based animation of rigid bodies. Knowing the current state of the rigid bodies and the derivative information at any time, a differential equation solver can now be used to compute the state vector at a subsequent time. Euler method, as given in Eq. (10), for example can be used but since it is unstable and inaccurate, more sophisticated methods such as Runge-Kutta method are appropriate. Although they are more computationally expensive than Euler method, large step sizes can be used resulting in an overall computational savings.

$$
\boldsymbol{x}(t+h)=\boldsymbol{x}(t)+h \cdot \dot{\boldsymbol{x}}(t)
$$

Finally, it is important to note that the sum of forces and sum of moments about rigid body's mass center in Eq. (9) include contact forces and collision forces (or impulses) when it moves relative to another rigid body. This of course entails the use of efficient collision detection routines. Since collision detection, response and contact handling are well documented in computer animation and robotics, it will not be discussed in detail in this paper.

\section{EARTHQUAKE GROUND MOTIONS}

Throughout the scope of this paper, the earthquake accelerogram used was that observed at Kobe Marine Meteorological Observatory during of the 1995 Kobe earthquake as shown in Fig. (2). The maximum accelerations in EW-, NS-, and UD-directions are $6.0 \mathrm{~m} / \mathrm{s}^{2}$ at $5.5 \mathrm{~s}, 8.2 \mathrm{~m} / \mathrm{s}^{2}$ at $5.5 \mathrm{~s}$, and $3.3 \mathrm{~m} / \mathrm{s}^{2}$ at $4.7 \mathrm{~s}$, respectively.
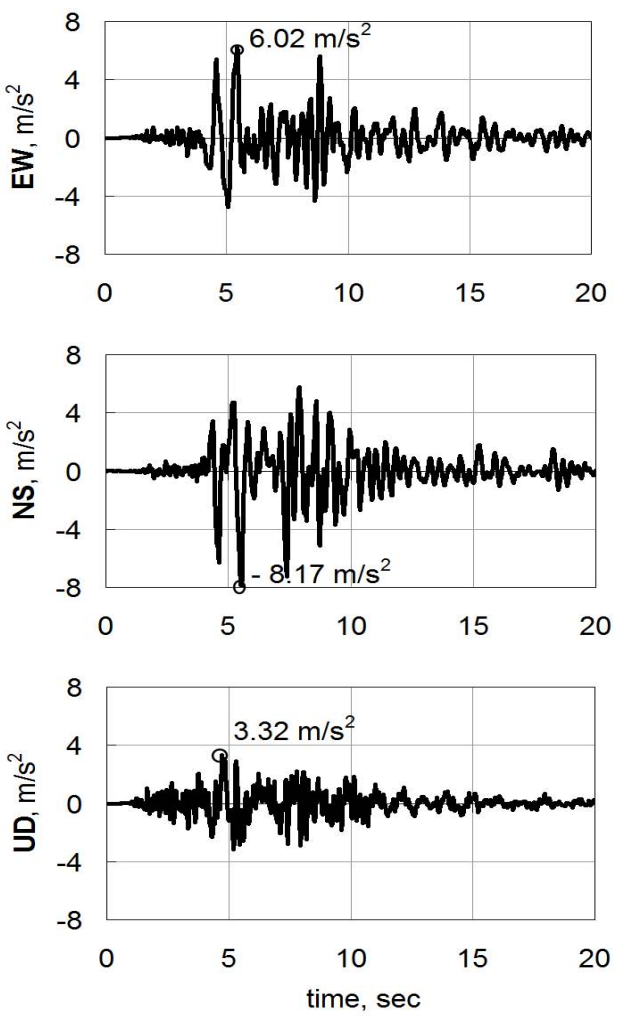

Fig. (2). Time history of ground acceleration of the 1995 Kobe earthquake used in analyses.

\section{SYSTEM OF NON-LINEAR SPRINGS AND DASH- POTS}

\section{Deformations and Restoring Forces of a Link}

A new link consisting of a spring and a damper, parallel to one another is introduced. Forces exerted to points on connected bodies are the vector sum of forces exerted by the spring and damper components. The stress-strain behavior of materials, as modeled by springs, is idealized by the straight lines in Fig. (3). In this model the restoring force in tension is proportional to the strain up to $\varepsilon_{Y T}$ with maximum yield restoring force $F_{Y T}$. The second straight line represents the strain-hardening characteristic until point $\mathrm{C}$, when the restoring force reaches its ultimate value $F_{U T}$. For different set of parameters $\varepsilon_{Y C}, F_{Y C}, \varepsilon_{U C}$, and $F_{U C}$, the behavior in compression can be modeled.

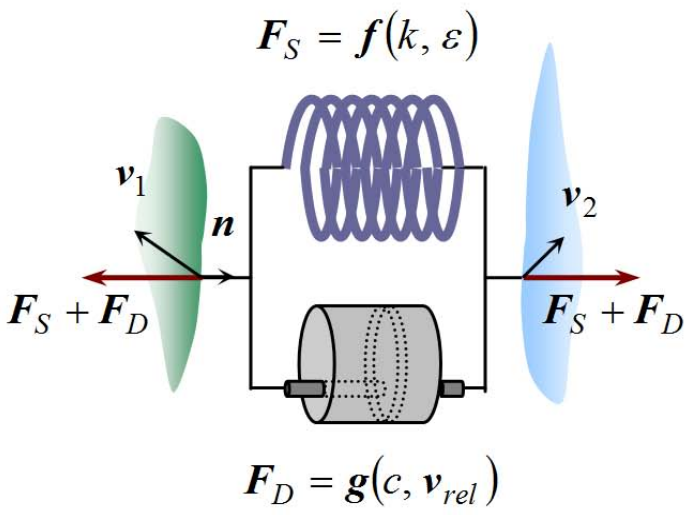

(a)

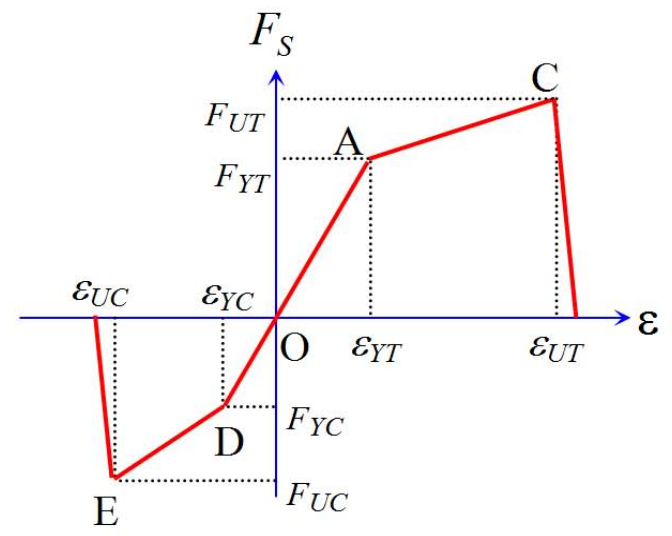

(b)

Fig. (3). Force exerted by a link on rigid bodies.

The restoring force exerted by the damper can be computed similarly for any relative velocity as $v_{r e l}=n \cdot\left(v_{1}-v_{2}\right)$ 
If the strain in the link exceeds its maximum value $\varepsilon_{U T}$ or $\varepsilon_{U C}$, no subsequent forces are exerted to the connected bodies. The link is marked DELETED to aid analysis of link failure.

\section{Proposed System of Inelastic Links}

To simulate inelastic behaviour of structural components, a configuration of nonlinear links are positioned such as shown in Fig. (4a).

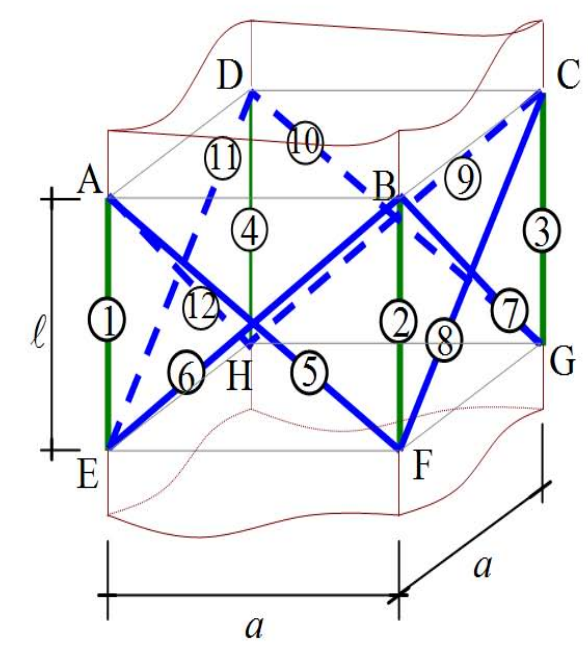

(a)

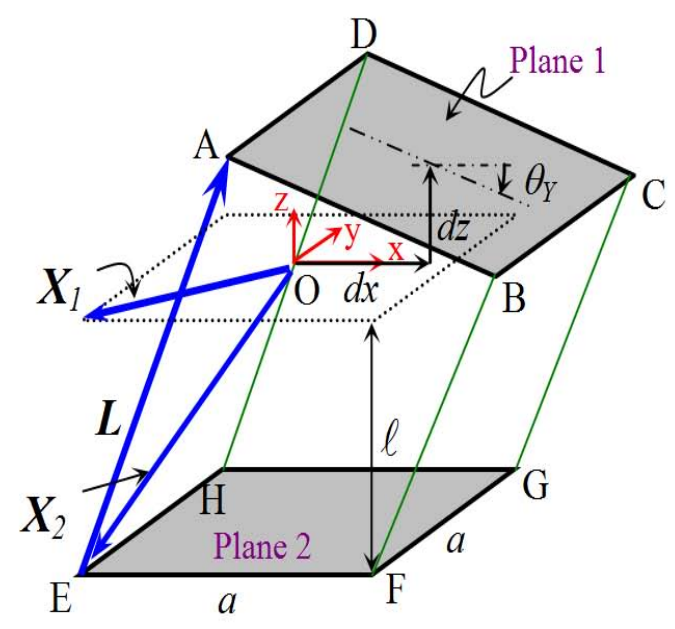

(b)

Fig. (4). Example of link system used to model plastic hinges; (a) initial configuration, (b) deformation of links due to in plane displacements $d x, d z$, and $\theta_{Y}$.

\section{DEFORMATIONS AND RESTORING FORCES OF LINK SYSTEM}

Fig. (4b) shows the deformation of the link system (diagonal links not shown) due to in-plane displacements $d x, d z$ and rotation about $y$-axis $\theta_{Y}$. The elongation $\delta$ of any spring can be computed by subtracting the original length $\left|\boldsymbol{L}_{0}\right|$ from the deformed length $|\boldsymbol{L}|$ of the spring where

$$
\boldsymbol{L}=\left(\left[\begin{array}{ccc}
\cos \theta_{Y} & 0 & \sin \theta_{Y} \\
0 & 1 & 0 \\
-\sin \theta_{Y} & 0 & \cos \theta_{Y}
\end{array}\right] \cdot \boldsymbol{X}_{1}+\left\{\begin{array}{c}
d x \\
0 \\
d z
\end{array}\right\}\right)-\boldsymbol{X}_{2}
$$

Here, $\boldsymbol{X}_{n}$ is the coordinates of the endpoint of the spring on plane $n$ in the undeformed configuration.

For spring numbered 1, we have

$$
\begin{aligned}
& \boldsymbol{X}_{1}=\boldsymbol{X}_{A}=\left\{\begin{array}{lll}
-a / 2 & -a / 2 & 0
\end{array}\right\}^{T} \text { and } \\
& \boldsymbol{X}_{2}=\boldsymbol{X}_{E}=\left\{\begin{array}{lll}
-a / 2 & -a / 2 & -\ell
\end{array}\right\}^{T}
\end{aligned}
$$

and therefore the deformed length vector $\boldsymbol{L}_{1}$ calculated using Eq. (11) becomes

$\boldsymbol{L}=\left\{\begin{array}{c}d x+\frac{a}{2}\left(1-\cos \theta_{Y}\right) \\ 0 \\ d z+\ell+\frac{a}{2} \sin \theta_{Y}\end{array}\right\}$

Due to the given spring configuration, the displacements would simply the same amount of elongation for springs 1 and 4 such that,

$$
\begin{aligned}
& \delta_{1}=|\boldsymbol{L}|-\left|\boldsymbol{L}_{0}\right| \\
& =\sqrt{\left[d x+\frac{a}{2}\left(1-\cos \theta_{Y}\right)\right]^{2}+\left[d z+\ell+\frac{a}{2} \sin \theta_{Y}\right]^{2}}-\ell \\
& =\delta_{4}
\end{aligned}
$$

Similarly for spring numbered 2 , the following expression can be derived,

$$
\delta_{2}=\sqrt{\left[d x-\frac{a}{2}\left(1-\cos \theta_{Y}\right)\right]^{2}+\left[d z+\ell-\frac{a}{2} \sin \theta_{Y}\right]^{2}}-\ell=\delta_{3}
$$

And for the diagonal springs, we have

$$
\begin{aligned}
\delta_{5}= & \sqrt{\left[d x-\frac{a}{2}\left(1-\cos \theta_{Y}\right)\right]^{2}+\left[d z+\ell+\frac{a}{2} \sin \theta_{Y}\right]^{2}} \\
- & \sqrt{a^{2}+\ell^{2}}=\delta_{10} \\
\delta_{6}= & \sqrt{\left[d x+\frac{a}{2}\left(1+\cos \theta_{Y}\right)\right]^{2}+\left[d z+\ell-\frac{a}{2} \sin \theta_{Y}\right]^{2}} \\
& -\sqrt{a^{2}+\ell^{2}}=\delta_{9} \\
\delta_{7}= & \sqrt{a^{2}+\left[d x-\frac{a}{2}\left(1-\cos \theta_{Y}\right)\right]^{2}+\left[d z+\ell-\frac{a}{2} \sin \theta_{Y}\right]^{2}} \\
& -\sqrt{a^{2}+\ell^{2}}=\delta_{8}
\end{aligned}
$$




$$
\begin{aligned}
\delta_{11}= & \sqrt{a^{2}+\left[d x+\frac{a}{2}\left(1-\cos \theta_{Y}\right)\right]^{2}+\left[d z+\ell+\frac{a}{2} \sin \theta_{Y}\right]^{2}} \\
& -\sqrt{a^{2}+\ell^{2}}=\delta_{12}
\end{aligned}
$$

The above set of equations are the expressions for the deformations of the links in Fig. (4b) for finite displacements $d x, d z$, and $\theta_{Y}$. Assuming small values of displacements $d x, d z$, and $\theta_{Y}$, these expressions can be simplified using the Taylor series expansion of $\delta_{i}=\delta_{i}\left(d x, d z, \theta_{Y}\right)$ about initial configuration $\left(d x, d z, \theta_{Y}\right)=(0,0,0)$. Here, we can neglect higher-ordered terms so that we have:

$$
\begin{aligned}
\delta_{i}\left(d x, d z, \theta_{Y}\right) \cong & \delta_{i}(0,0,0)+\left.d x \frac{\partial \delta_{i}}{\partial d x}\right|_{(0,0,0)} \\
& +\left.d z \frac{\partial \delta_{i}}{\partial d z}\right|_{(0,0,0)}+\left.\theta_{Y} \frac{\partial \delta_{i}}{\partial \theta_{Y}}\right|_{(0,0,0)}
\end{aligned}
$$

Assuming that all springs are initially undeformed, i.e. $\delta_{i}(0,0,0)=0$ for $i=1 \ldots 12$, Eqs. (14)-(16) reduce to the expressions for infinitesimal displacements $d x, d z$, and $\theta_{Y}$,

$$
\begin{aligned}
& \delta_{1}=d z+\frac{a \theta_{Y}}{2}=\delta_{4} \\
& \delta_{2}=d z-\frac{a \theta_{Y}}{2}=\delta_{3} \\
& \delta_{5}=\frac{-2 a d x+2 \ell d z+a \ell \theta_{Y}}{2 \sqrt{a^{2}+\ell^{2}}}=\delta_{10} \\
& \delta_{6}=\frac{2 a d x+2 \ell d z-a \ell \theta_{Y}}{2 \sqrt{a^{2}+\ell^{2}}}=\delta_{9} \\
& \delta_{7}=\frac{2 \ell d z-a \ell \theta_{Y}}{2 \sqrt{a^{2}+\ell^{2}}}=\delta_{8} \\
& \delta_{11}=\frac{2 \ell d z+a \ell \theta_{Y}}{2 \sqrt{a^{2}+\ell^{2}}}=\delta_{12}
\end{aligned}
$$

\section{Pure Axial Deformation}

A special case of deformation is when plane ABCD moves an infinitesimal distance $d z$ relative to plane $\mathrm{EFGH}$, as in Fig. (5a). The expression for the strains at each spring, with deformations obtained from Eq. (18) by setting $d x=0$ and $\theta_{Y}=0$,

$$
\begin{aligned}
& \varepsilon_{1}=\varepsilon_{2}=\varepsilon_{3}=\varepsilon_{4}=\frac{d z}{\ell} \\
& \varepsilon_{5}=\varepsilon_{6}=\varepsilon_{7}=\varepsilon_{8}=\varepsilon_{9}=\varepsilon_{10}=\varepsilon_{11}=\varepsilon_{12}=\frac{\ell \cdot d z}{a^{2}+\ell^{2}}
\end{aligned}
$$

the resultant restoring force $P_{T}$ becomes

$$
P_{T}=4 F_{1 Z}+8 F_{5 Z}=4 k \cdot d z+\frac{8 k^{\prime} \ell^{2}}{a^{2}+\ell^{2}} d z
$$

where $k$ and $k^{\prime}$ are spring constants for axial and diagonal springs, respectively.

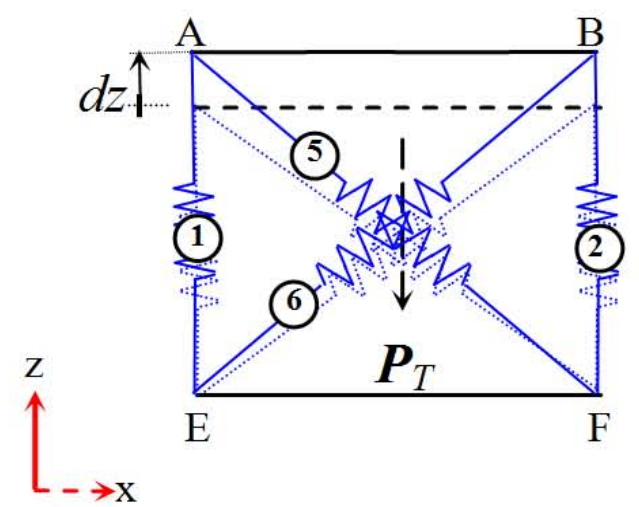

(a)

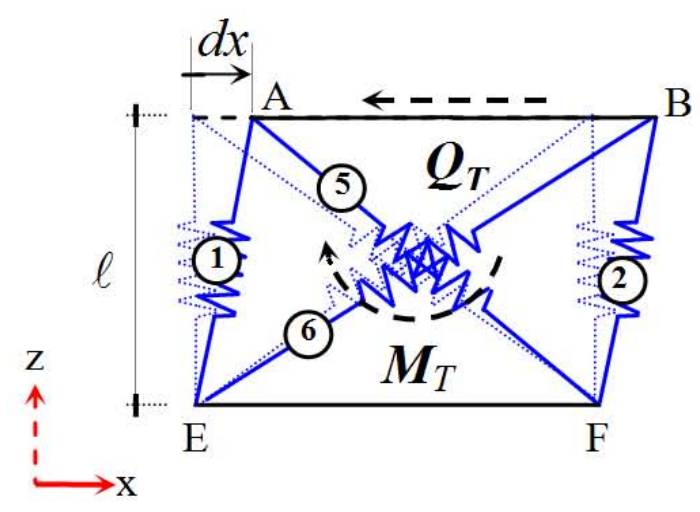

(b)

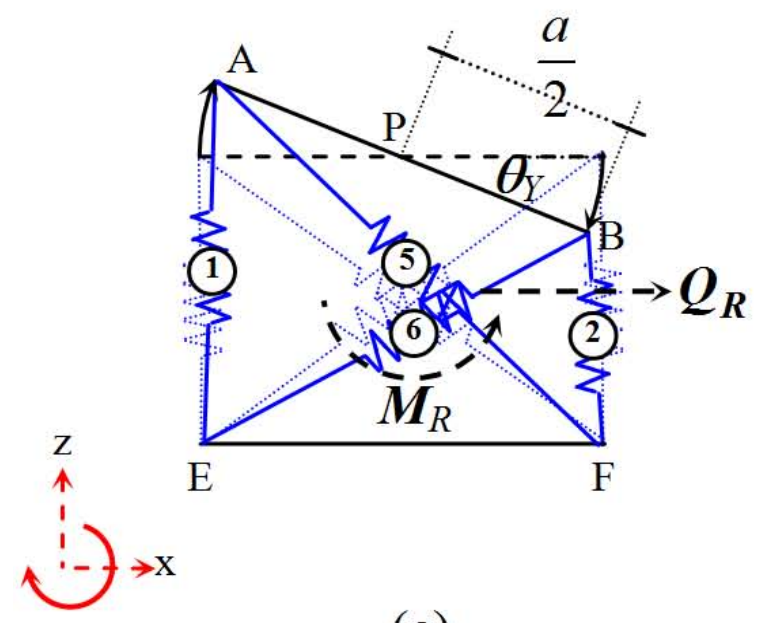

(c)

Fig. (5). Special cases of link deformations.

\section{Pure Shear Deformation}

Another special case of deformation is when plane ABCD moves an infinitesimal displacement $d x$ relative to plane EFGH, as in Fig. (5b). The strain on the springs, with 


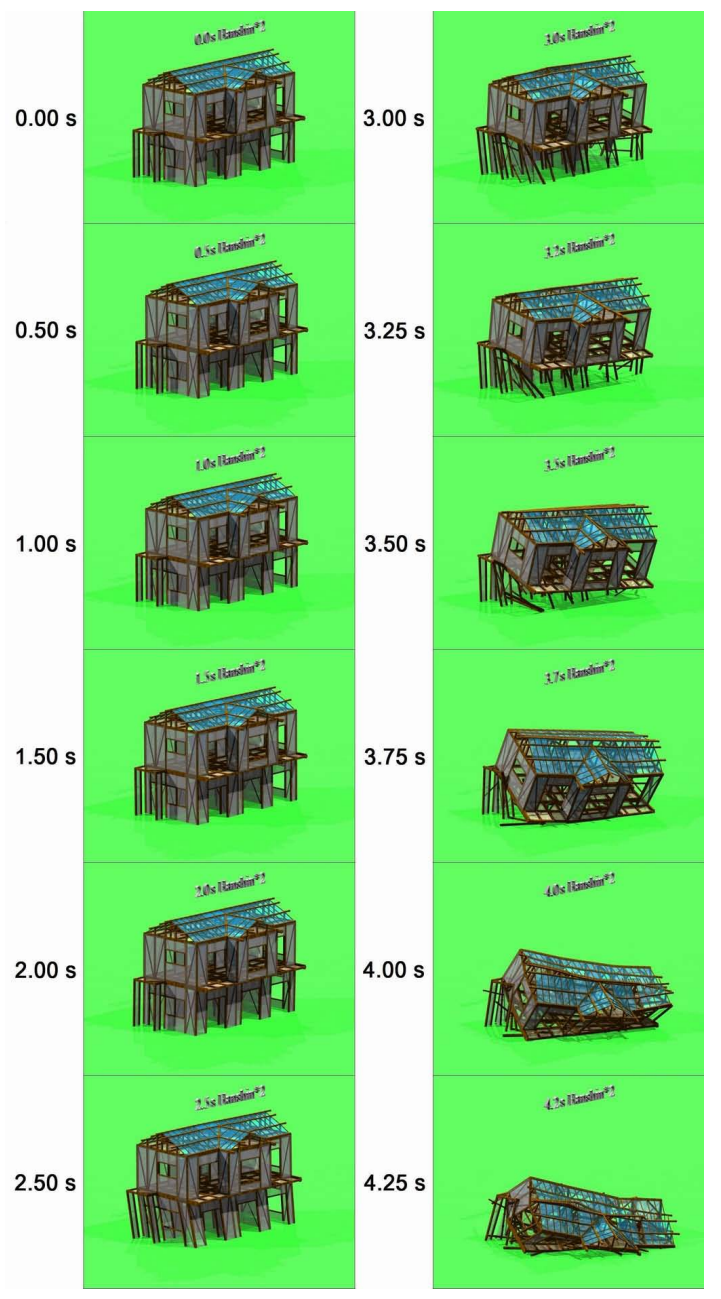

$$
\begin{aligned}
Q_{T} & =4 F_{5 X}=4 \cdot\left(k^{\prime} \frac{-a \cdot d x}{\sqrt{a^{2}+\ell^{2}}} \cdot \frac{a}{\sqrt{a^{2}+\ell^{2}}}\right) \\
& =-\frac{4 a^{2} k^{\prime}}{a^{2}+\ell^{2}} d x \\
M_{T} & =2 F_{6 Z} \cdot a=\frac{2 a^{2} k^{\prime} \ell}{a^{2}+\ell^{2}} \cdot d x
\end{aligned}
$$

\section{Pure Bending Deformation}

When plane $\mathrm{ABCD}$ rotates a small angle $\theta_{Y}$ about an axis passing through $\mathrm{P}$ and perpendicular to the plane of the paper, as shown in Fig. (5c), the elongations of springs are obtained from Eq. (18) by setting $d x=d z=0$ so that the strains at each spring become

$$
\begin{aligned}
& \varepsilon_{1}=\varepsilon_{4}=-\varepsilon_{2}=-\varepsilon_{3}=\frac{a}{2 \ell} \theta_{Y} \\
& \varepsilon_{5}=\varepsilon_{10}=\varepsilon_{11}=\varepsilon_{12}=\frac{a \ell}{2\left(a^{2}+\ell^{2}\right)} \theta_{Y}=-\varepsilon_{6}=-\varepsilon_{7}=-\varepsilon_{8}=-\varepsilon_{9}
\end{aligned}
$$

The resultant restoring force $Q_{T}$ and the moment $M_{T}$ are

$$
\begin{aligned}
Q_{R} & =4 F_{5 X}=4 \cdot\left(\frac{a k^{\prime} \ell \theta_{Y}}{2 \sqrt{a^{2}+\ell^{2}}} \cdot \frac{a}{\sqrt{a^{2}+\ell^{2}}}\right)=\frac{2 a^{2} k^{\prime} \ell}{a^{2}+\ell^{2}} \theta_{Y} \\
M_{R} & =2 F_{2} \cdot a+2 F_{6 Z} \cdot a+2 F_{8 Z} \cdot a \\
& =a^{2} k \theta_{Y}+\frac{2 a^{2} k^{\prime} \ell^{2}}{a^{2}+\ell^{2}} \theta_{Y}
\end{aligned}
$$

\section{Effective Stiffness in Axial, Shear and Bending Deforma- tions}

From Eqs. (20), (22) and (26) we have the following effective stiffness in axial, shear, and bending as

$$
\begin{aligned}
& K_{P}=4 k+\frac{8 \ell^{2}}{a^{2}+\ell^{2}} k^{\prime} \\
& K_{V}=\frac{4 a^{2}}{a^{2}+\ell^{2}} k^{\prime} \\
& K_{M}=a^{2} k+\frac{2 a^{2} \ell^{2}}{a^{2}+\ell^{2}} k^{\prime}
\end{aligned}
$$

Fig. (7). A reinforcement plan of the wooden house.

deformations obtained from Eq. (18) where $d z=\theta_{Y}=0$, and the resultant restoring force $Q_{T}$ and $M_{T}$ at the final configuration are:

$$
\begin{aligned}
& \varepsilon_{1}=\varepsilon_{2}=\varepsilon_{3}=\varepsilon_{4}=\varepsilon_{7}=\varepsilon_{8}=\varepsilon_{11}=\varepsilon_{12}=0 \\
& \varepsilon_{5}=\varepsilon_{10}=-\varepsilon_{6}=-\varepsilon_{9}=\frac{-a \cdot d x}{a^{2}+\ell^{2}}
\end{aligned}
$$

respectively for the link configuration.

\section{APPLICATION TO WOODEN HOUSES}

\section{Dynamic Collapse of Conventional Wooden Houses}

Using the numerical method presented above, earthquake responses of typical wooden houses in Japan were computed. In modeling, the dead load of the floor slab, beams, columns, walls and roofs, and the live loads were estimated. The stress-strain curves for the material of the structural members were modeled. The model structures were then subjected to 
doubly-amplified waves of the 1995 Kobe earthquake in Fig. $\mathbf{2}$, and various collapse mechanisms of wooden houses were observed including (a) collapse due to the soft first story, (b) tumbling type collapse, (c) failure at the second floor due to the amplification of the vibration at the upper floor, and (d) collapse of intermediate floor.

Even though the soft first story type of collapse is often stressed, the failure mechanism depends on the design and physical layout of the structure, i.e., strength and distributions of columns, beams and walls. For example, Fig. (6) [4] shows the response sequence of the two-storey wooden house. During the strong motion earthquake, the house deformed beyond the limit of linearly elastic behavior, and the southern portion of the first floor began to fail at around $3 \mathrm{~s}$, and collapsed at about $4 \mathrm{~s}$.

In order to avoid the collapse in Fig. (6), several retrofit plans were developed such as shown in Fig. (7), where the red members indicate added columns and diagonal braces. The method proposed in this paper was then used to simulate the earthquake response of each of the reinforced houses. Results showed that the house in Fig. (7) withstood the same earthquake motions used in Fig. (6), i.e., the doublyamplified waves of the 1995 Kobe earthquake. Based on the obtained results, engineers can propose retrofit plans so house owners can improve the performance of their houses against earthquakes.

\section{RELIABILITY OF SIMULATED RESPONSE}

Most wooden houses in Japan are generally composed of frame units made of columns and beams, and walls. Therefore, in order to establish the reliability and correctness of collapse simulation of an entire house, it is obligatory to show the agreement of simulated and experimental results for such structural units. Fig. (8) shows frames with different types of reinforcements. A monotonically increasing force was then applied horizontally to the upper beam of each frame, and the response was computed using the same program that was used in Fig. (6).

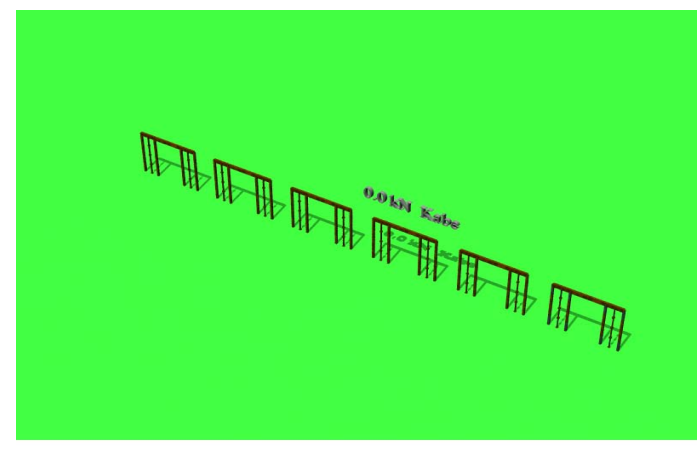

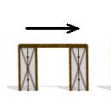

(6)

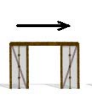

(5)

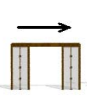

(4)

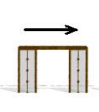

(3)

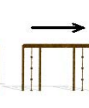

(2)

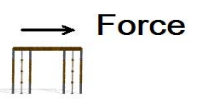

(1)
Fig. (8). Samples of wooden frame units. From right to left: wooden frames with (1) no end connection plate; (2) end connection plates; (3) plaster board; (4) siding board; (5) two single braces, plaster board and siding board; and (6) two double braces, plaster board and siding board.
Based on the obtained response of the upper beam, the relationship between the displacement and force was plotted by solid lines in Fig. (9) for each frame unit (1) through (6). The experimental relationships obtained by Miyoshi et al. [5] were also plotted by dashed lines in Fig. (9). The numerical and the experimental curves agree well including the rigidity in small strain, the maximum strength, and the weakening process, and this shows the reliability of the simulation in this paper.

\section{CONCLUSIONS}

This research attempted to simulate seismic collapse of wooden houses subjected to the 1995 Kobe earthquake using the Rigid Body-Spring Method (RBSM) for the purpose of retrofitting. The following conclusions can be drawn:

1. The proposed method is capable of demonstrating to some extent various collapse behavior of wooden houses during strong motion earthquakes. More accurately, the link system used to characterize plastic hinges of the simplified structural components can simulate local failure that causes the entire house to collapse during strong motion earthquakes.

2. The method provides a way to identify the weak point of a structure thus allowing engineers to perform retrofitting analysis easily and to suggest ways to improve the seismic performance of built wooden houses.

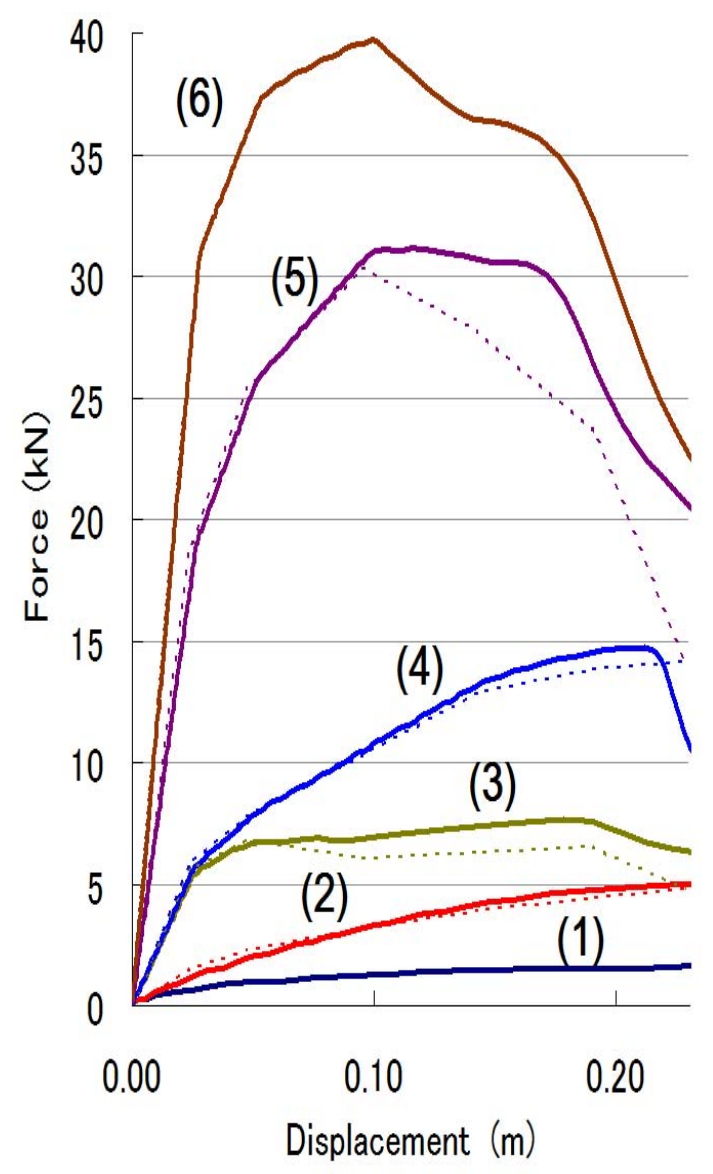

Fig. (9). Force-displacement relationships of wooden frames. 


\section{REFERENCES}

[1] T. Kawai. "New element models in discrete structural analysis". Japan Society of Naval Architects, Japan; vol. 141, pp. 174-180, 1977.

[2] D. Baraff. Dynamic simulation of non-penetrating rigid bodies. Ph.D. Thesis, Cornell University; 1992.

[3] W.R. Hamilton. "On quaternions". Proc R Irish Acad; vol. 3, pp. 1$16,1847$.
[4] H. Kawakami. http://www.saitama-u.ac.jp/ kawakami/ (in Japanese).

[5] K. Miyoshi, Y. Ohashi, K. Takahashi, M. Watahiki and I. Nakano. "Static loading test and shaking table test of walls for wooden houses, Part 1 static loading test". Summaries of Technical Papers of Annual Meeting, Architectural Institute of Japan 2001: pp. 199200 (in Japanese).

(C) Tingatinga et al:: Licensee Bentham Open.

This is an open access article licensed under the terms of the Creative Commons Attribution Non-Commercial License (http://creativecommons.org/licenses/by-nc/3.0/) which permits unrestricted, non-commercial use, distribution and reproduction in any medium, provided the work is properly cited. 